$$
\alpha_{0}(\omega)=\frac{2\left|\left\langle\Psi_{0}\left|Q_{l}^{m}\right| \tilde{\Phi}\right\rangle\right|^{2}\left\langle\tilde{\Phi}\left|H-E_{0}\right| \tilde{\Phi}\right\rangle}{\left\langle\tilde{\Phi}\left|H-E_{0}\right| \tilde{\Phi}\right\rangle^{2}-\omega^{2}\langle\tilde{\Phi} \mid \tilde{\Phi}\rangle},
$$

such that (13) inserted into the standard formula

$$
C_{6}=\frac{3}{\pi} \int|\alpha(i \omega)|^{2} d \omega
$$

yields exactly the best lower bound (12).
${ }^{1}$ See, e.g., J. O. Hirschfelder, W. Byers-Brown, and S. T. Epstein, Adv. Quantum Chem. (to be published).

${ }^{2}$ P. O. Löwdin, Phys. Rev. 139, A357 (1965); J. Chem. Phys. 43, S175 (1965).

${ }^{3} \mathrm{~W}$. Kutzelnigg and $\mathrm{F}$. Maeder (to be published).

${ }^{4}$ H. L. Kramer, J. Chem. Phys. 53, 2783 (1970).

${ }^{5}$ R. T Pack, Chem. Phys. Lett. 5, 257 (1970).

${ }^{6} \mathrm{~F}$. Maeder and W. Kutzelnigg (to be published).

${ }^{7}$ W. Kutzelnigg, Chem. Phys. Lett. 4, 435 (1969).

\title{
Search for polarized photoelectrons from molecules
}

\author{
U. Heinzmann, J. Kessier, and E. Kuhlmann \\ Physikalisches Institut der Universität Münster, Münster, Germany \\ (Received 3 February 1978)
}

One of the novel results in photon-atom interaction is the experimental evidence for spin polarization of photoelectrons produced by circularly polarized light. After this effect had been verified for free alkali atoms for which it has originally been predicted, ${ }^{1}$ experimental studies were made with other targets and positive results were obtained with all the substances studied so far (for a summary see Ref. 2).

The interest in this phenomenon comes mainly from the fact that such studies give information on details of the photoionization process (e.g., the influence of spinorbit interaction on the cross sections for transitions into different partial continua ${ }^{2,3}$ ). In certain favorable cases such processes can also be utilized as sources of polarized electrons. 4

One of the first steps on the way to a profound understanding of this field must be the attempt to get a survey of the materials that are particularly suitable candidates for yielding polarized electrons when photoionized with circularly polarized light. From the positive results obtained both with free atoms and solids and from their interpretation it seems very likely that also several molecules will yield polarized photoelectrons.

The present Communication reports on first experimental studies in this direction. The selection of the molecules to be used was mainly determined by the following conditions: The experiment was to be made with an apparatus which had been used for polarization measurements on photoelectrons from nonalkalis..$^{3,5}$ In this apparatus circularly polarized vuv radiation of wavelengths longer than $140 \mathrm{~nm}$ could be produced. Consequently, the experiments were restricted to molecules with ionization energies below $8.8 \mathrm{eV}$. Further, the photoionization cross sections should not be too small $\left(>10^{-18} \mathrm{~cm}^{2}\right)$ since photoelectron currents of more than $5 \times 10^{-17} \mathrm{~A}$ were needed for polarization analysis in our high-efficiency Mott detector. Needless to say, only such substances were appropriate which could be handled in a vacuum apparatus and could be evaporated to form a reasonable molecular vapor beam.

In addition to these more technical considerations one has to raise the crucial question of which molecules are likely to yield an appreciable polarization of the photoelectrons. From the experience made so far with atoms and solids one can say quite generally that a large spinorbit interaction in the system studied and a pronounced structure of the photoionization cross section favor high photoelectron polarization. Apart from these rather general criteria no specific information was available to make possible an estimate of the polarization to be expected: A theoretical treatment of this problem for molecular targets has not yet been made and a quantitative explanation of the mechanism by which the photon spin is transferred to the photoelectron spin must certainly be different for atoms and for molecules. This becomes evident when one remembers that the calculation of the photoelectron polarization in the case of atoms takes advantage of the possibility of assigning well-defined angular momenta to the electrons, which no longer works for molecules which do not possess spherical symmetry.

There is, however, one feature known from atoms with closed $p$ subshells which is likely to occur also in the case of molecules with closed $\pi$ orbitals: It has been theoretically predicted ${ }^{6}$ for rare gas atoms that the photoelectron polarization has different signs for transitions leaving the ion in neighboring doublet states so that one obtains zero polarization if this fine structure is not resolved. That is why we made the bandwidth $\Delta \lambda$ of the light as small as was compatible with the intensities to be at least obtained. We worked at the photoionization threshold in order to be able to resolve the fine-structure splitting in favorable cases; an electron spectrometer for separation of photoelectrons with different energies has not been used. Most of the measurements were made at the intensity maximum of the $\mathrm{H}_{2}$ discharge lamp, $\lambda=160.8 \mathrm{~nm} \hat{=} h \nu=7.71 \mathrm{eV}$. Since aniline has an (adiabatic) ionization energy of $7.65 \mathrm{eV}$ and also fulfills the other conditions discussed above the measurements were concentrated on this molecule. We also studied the derivatives 2-methylaniline, 2,6dimethylaniline, $N N$-dimethylaniline, and 3-iodoaniline having (adiabatic) ionization energies between $7.1 \mathrm{eV}$ and less than $7.7 \mathrm{eV} .^{7}$ Iodoaniline has been used be- 
TABLE I. Experimental results for polarization of photoelectrons ejected by circularly polarized vuv radiation from molecular targets.

\begin{tabular}{lcc}
\hline \hline Molecule & $\begin{array}{c}\text { Wavelength } \\
\text { half-bandwidth }(\mathrm{nm})\end{array}$ & $\begin{array}{c}\text { Polarization } \pm \text { single } \\
\text { statistical error }\end{array}$ \\
\hline Aniline & $160.8 \pm 0.25$ & $-0.03 \pm 0.04$ \\
2-Methylaniline & $160.8 \pm 0.6$ & $0.00 \pm 0.02$ \\
2,6-Dimethylaniline & $160.8 \pm 0.3$ & $-0.04 \pm 0.07$ \\
NA-Dimethylaniline & $160.8 \pm 0.15$ & $-0.01 \pm 0.04$ \\
NN-Dimethylaniline & $158.0 \pm 0.8$ & $-0.01 \pm 0.02$ \\
3-Iodoaniline & $160.8 \pm 1.5$ & $-0.03 \pm 0.18$ \\
Tellurium & $160.8 \pm 1.5$ & $0.01 \pm 0.07$ \\
Tellurium & $149.4 \pm 1.5$ & $-0.01 \pm 0.09$ \\
\hline \hline
\end{tabular}

cause the heavy iodine is supposed to increase the spinorbit interaction in this molecule. For similar purposes we studied $\mathrm{Te}_{2}$ where the abovementioned doublet splitting of the ion is known $^{8,9}$ to be $470 \mathrm{meV}(\hat{=} 8 \mathrm{~nm})$ which could be easily resolved in our experiment.

The measurements have been performed with the apparatus described earlie $r^{5}$ after having made a few minor modifications. For evaporation of the anilines a new oven has been constructed taking account of the physical and chemical properties of these substances. The oven was heated to $\sim 350 \mathrm{~K}$ (420 K for iodoaniline) so that vapor pressures of about 10 torr were obtained therein. Tellurium (purity 99.6\% with the impurities $\mathrm{Cl}<0.05 \%$, Se and $\mathrm{S}<0.01 \%, \mathrm{Mg}, \mathrm{Pb}, \mathrm{Al}, \mathrm{Si}, \mathrm{Fe}$, and $\mathrm{Ca}<0.005 \%$ ) was evaporated at $940 \mathrm{~K}$ from the oven used in the former experiments with thallium and lead. According to Ref. 8 the tellurium vapor consists of $99 \%$ $\mathrm{Te}_{2}$ and about $1 \%$ other tellurium molecules.

In order to make sure that the apparatus was reliable, test measurements of the photoelectron polarization with thallium atoms have been made; they confirmed our earlier results. ${ }^{3}$ However, with none of the molecules studied has a polarization been found different from zero. The results are listed in Table $I$. The large values for the bandwidth $\Delta \lambda$ and for the error of the electron polarization in the case of iodoaniline are caused by difficulties in evaporating this substance which allowed only small target densities to be produced. The fact that in the case of tellurium even at $\lambda=160.8 \mathrm{~nm}$ photoelectrons were obtained is not what we had expected from the results of Ref. 8 where

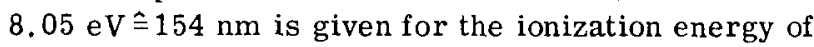
$\mathrm{Te}_{2}$. A possible explanation could be that under the conditions of the present experiment an appreciable percentage of tellurium molecules other than $\mathrm{Te}_{2}$ is formed. (At $160.8 \mathrm{~nm}$ about ten times the light intensity was needed to produce the photoelectron rate obtained at $149.4 \mathrm{~nm}$.)

It is easy to list a handful of arguments (unsuitable molecules or wavelengths, $\Delta \lambda$ too large, etc.) why no photoelectron polarization has been found in the present measurements. Because of the rudimentary stage of theory and the limited results of this very first experiment, this would, however, not be far from speculation so that we will refrain from it. Hope for further experimental progress is raised by more versatile vuv sources, like synchrotron radiation, which we are going to apply in experiments of similar kind.

We are grateful to Dr. G. Wünsch, Institute of Anorganic Chemistry, for analysis of the tellurium. Equipment made available by the Deutsche Forschungsgemeinschaft has been used in this investigation.

${ }^{1} \mathrm{U}$. Fano, Phys. Rev. 178, 131 (1969).

${ }^{2} \mathrm{~J}$. Kessler, Polarized Electrons (Springer-Verlag, BerlinHeidelberg- New York, 1976).

${ }^{3} \mathrm{U}$. Heinzmann, H. Heuer, and J. Kessler, Phys. Rev. Lett. 36, 1444 (1976).

${ }^{4}$ D. T. Pierce and F. Meier, Phys. Rev. B 13, 5484 (1976). ${ }^{5}$ U. Heinzmann, J. Phys. E 10, 1001 (1977); J. Phys. B 11 (1978) (in press).

${ }^{6}$ N. A. Cherepkov, Sov. Phys. -JETP 38, 463 (1974).

${ }^{7}$ J. P. Maier and D。W. Turner, J. Chem. Soc. F 2, 521 (1973).

${ }^{8}$ J. Berkowitz, J. Chem. Phys. 62, 4074 (1975).

${ }^{9}$ D. G. Streets and J. Berkowitz, J. Electron Spectrosc. 9, 269 (1976).

\title{
NOTES
}

\section{Lifetime measurements of $A^{3} \Pi_{1}$ vibrational levels of $\mathrm{ICl}^{\mathrm{a})}$}

\author{
M. D. Havey and J. J. Wright \\ Department of Physics, University of New Hampshire, Durham, New Hampshire 03824 \\ (Received 29 December 1977)
}

Although there have been extensive spectroscopic studies of the $A^{3} \Pi_{1}$ state of $I C 1,^{1-5}$ a discrepancy still exists regarding perturbations of various vibrational levels. Absorption measurements by Darbyshire ${ }^{1}$ and by Curtis and Patkowski ${ }^{2}$ indicate perturbations at about $v^{\prime}=9$ or 10 . Later work by Hulthén et al ${ }^{3, B}$ does not confirm this but contends there are perturbations for levels below $v^{\prime}=6$. Such perturbations, if they exist, 